\title{
Structural Arrangements and Bonding Analysis of $\mathrm{MgB}_{2} \mathrm{C}_{2}$
}

\author{
Dae-Bok Kang \\ Department of Chemistry, Kyungsung University, Busan 608-736, Korea. E-mail: dbkang@ks.ac.kr \\ Received April 13, 2010, Accepted July 26, 2010
}

\begin{abstract}
The orthorhombic $\mathrm{MgB}_{2} \mathrm{C}_{2}$ structure contains well-separated parallel graphite-like $\mathrm{B}_{2} \mathrm{C}_{2}{ }^{2-}$ layers which extend infinitely in two dimensions. Three possible ways to distribute $\mathrm{B}$ and $\mathrm{C}$ atoms in the hexagonal sublattice sites are adopted. Band structures for the hypothetical distribution patterns are examined to assess the electronic stability of these phases and to account for the observed arrangement by means of extended Hückel tight-binding calculations. The preferred choice is the layer with $\mathrm{B}$ and $\mathrm{C}$ alternating strictly so that $\mathrm{B}$ is nearest neighbor to $\mathrm{C}$ and vice versa. A rationale for this is given. Due to the alternation of $\mathrm{B}$ and $\mathrm{C}$ within the honeycomb layers, $\mathrm{MgB}_{2} \mathrm{C}_{2}$ is a band insulator, which through partial substitution of $\mathrm{Mg}$ with $\mathrm{Li}$, is predicted to turn metallic with holes in the $\sigma$ bands at the Fermi level.
\end{abstract}

Key Words: Coloring problem, Electronic structure, Extended Hückel calculations, Ternary metal borocarbides

\section{Introduction}

The discovery of superconductivity in $\mathrm{MgB}_{2}\left(T_{\mathrm{c}}=39 \mathrm{~K}\right)$ has stimulated strong interest in the layered compounds which are structurally similar to $\mathrm{MgB}_{2}{ }^{1,2}$ The structure of $\mathrm{MgB}_{2}$ contains hexagonal layers of graphite-like sheets of $\mathrm{B}$ atoms intercalated with $\mathrm{Mg}$ planes. $\mathrm{MgB}_{2} \mathrm{C}_{2}$ is a large gap insulator that has a crystal structure comparable to $\mathrm{MgB}_{2}$, with boron and carbon atoms alternately occupying the sites within the hexagonal graphitic layers. ${ }^{3}$ Possible superconductivity at low temperature has been reported for doped $\mathrm{MgB}_{2} \mathrm{C}_{2}$, but no convincing evidence for superconductivity has been established so far. ${ }^{4}$

The $\mathrm{MgB}_{2} \mathrm{C}_{2}$ structure consists of the stacking of two-dimensional (2D) B/C layers, with metal atoms in between. Although the properties of this family of metal borocarbides have been widely investigated, there is still an open question concerning their actual structures, especially with respect to the position of $\mathrm{B}$ and $\mathrm{C}$ atoms. The question of boron $v s$. carbon distribution within the layers, frequently termed the "coloring problem", is often faced by solid-state chemists. With three $\mathrm{B}$ and three $\mathrm{C}$ atoms to form hexagons in the $\mathrm{B}_{2} \mathrm{C}_{2} 6^{3}$ net of $\mathrm{MgB}_{2} \mathrm{C}_{2}$, three simple different $\mathrm{B} / \mathrm{C}$ topologies are possible within the sheet (see 1). X-ray diffraction analysis by Wörle and $\mathrm{Nesper}^{3}$ has shown that $\mathrm{MgB}_{2} \mathrm{C}_{2}$ adopts a coloring I with alternating boron and carbon atoms, consistent with our calculations leading to the conclusion that for this formal dianionic charge coloring I is more stable than the other distribution variants II and III which necessarily all have C-C and B-B bonds. Indeed, coloring I exhibits a band gap at the Fermi level $\left(\mathrm{E}_{\mathrm{f}}\right)$, whereas the other colorings II and III do not.

We report herein the qualitative theoretical analysis of the structural preference of $\mathrm{MgB}_{2} \mathrm{C}_{2}$ phase. Our theoretical approach to estimating the relative stability of the models based on the $6^{3}$ nets is supported by the agreement between the most stable model structure (i.e., coloring I) and the experimentally characterized structure. As a computational tool we use extended Hückel tight-binding (EHTB) band calculations, with parameters given in Table 1 . In what follows we present a detailed analysis of the band structures of the ideal $\mathrm{B}_{2} \mathrm{C}_{2}{ }^{2-} 6^{3}$ nets in which the $\mathrm{B}$ and $\mathrm{C}$ atoms form a planar honeycomb layer. The electronic structure was calculated for three model $\mathrm{B}_{2} \mathrm{C}_{2}$ sheets. Each Fermi level was shifted to the position corresponding to $\left[\mathrm{B}_{2} \mathrm{C}_{2}\right]^{2-}$.

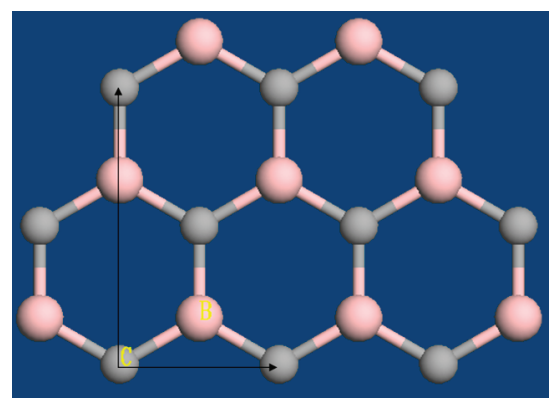

Coloring I

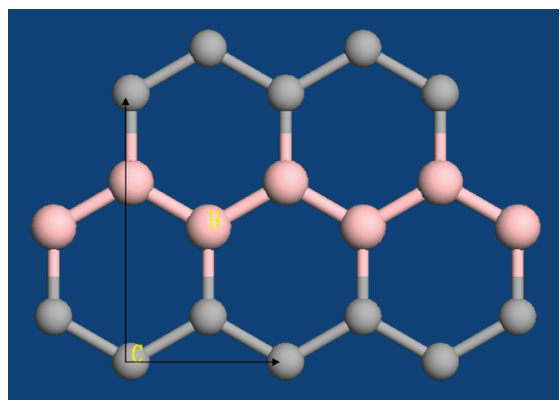

Coloring II

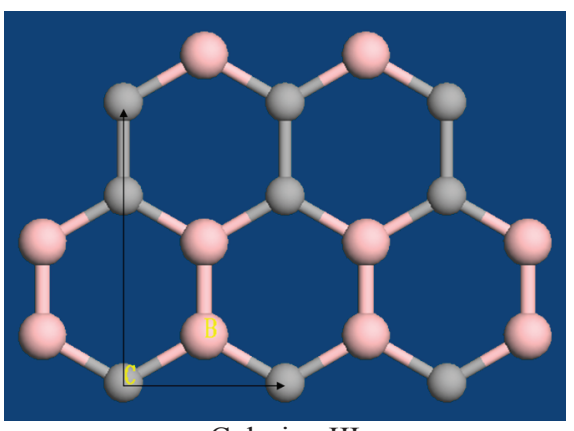

Coloring III 
Table 1. Atomic parameters used in the calculations

\begin{tabular}{cccc}
\hline atom & orbital & $\mathrm{H}_{\mathrm{ii}}, \mathrm{eV}$ & $\zeta$ \\
\hline $\mathrm{B}$ & $2 \mathrm{~s}$ & -15.2 & 1.30 \\
& $2 \mathrm{p}$ & -8.5 & 1.30 \\
$\mathrm{C}$ & $2 \mathrm{~s}$ & -21.4 & 1.625 \\
& $2 \mathrm{p}$ & -11.4 & 1.625 \\
$\mathrm{Mg}$ & $3 \mathrm{~s}$ & -9.0 & 1.10 \\
& $3 \mathrm{p}$ & -4.5 & 1.10 \\
\hline
\end{tabular}

Since the existence of holes in the $\sigma$ bands contributes to superconductivity in $\mathrm{MgB}_{2},{ }^{6}$ attempts have been made to achieve the same result by doping $\mathrm{MgB}_{2} \mathrm{C}_{2}$. So far no characteristics associated with a superconducting transition have been observed in Li-doped $\mathrm{MgB}_{2} \mathrm{C}_{2}$ at low temperatures. ${ }^{4}$ In this paper, we present results for the electronic structure of $\mathrm{Mg}_{1-x} \mathrm{Li}_{x} \mathrm{~B}_{2} \mathrm{C}_{2}$ $(x=0.125)$ and compare it with that of $\mathrm{MgB}_{2}$. Band structure calculations of $\mathrm{Mg}_{1-x} \mathrm{Li}_{x} \mathrm{~B}_{2} \mathrm{C}_{2}$ show that the Fermi level moves into the valence band as $x$ increases. Our rigid-band model calculations for Li-doped compounds predict that introducing nonstoichiometric Li could give rise to a finite density of states at the Fermi level and make the system metallic. Once it is holedoped, the states at the Fermi level include $\sigma$ bands, nearly flat along the direction perpendicular to the $\mathrm{BC}$ hexagonal layers, with an elliptical 2D Fermi surface. The calculated electronic states also show a remarkable similarity between the orbitals near the Fermi level in $\mathrm{MgB}_{2}$ and doped $\mathrm{MgB}_{2} \mathrm{C}_{2}$. There are equivalent hole pockets in the energy bands for both compounds. Thus, it is the peculiar molecular orbitals at the Fermi level that appear to be crucial to the high- $\mathrm{T}_{\mathrm{c}}$ superconductivity. The highest occupied bands consist of $\sigma\left(2 p_{x}\right.$ and $\left.2 p_{y}\right)$ orbitals on boron and carbon sites within the BC layer.

\section{Structure of $\mathrm{MgB}_{2} \mathrm{C}_{2}$}

The orthorhombic unit cell of $\mathrm{MgB}_{2} \mathrm{C}_{2}{ }^{3}$ is shown in Figure 1. The borocarbide $\mathrm{MgB}_{2} \mathrm{C}_{2}$ is isoelectronic and structurally similar to $\mathrm{MgB}_{2}$. The structure contains isolated two-dimensional (2D) $\mathrm{B}_{2} \mathrm{C}_{2}{ }^{2-}$ layers which obviously resemble graphite. The three-dimensional structure can be viewed as a series of layers alternately containing the $\mathrm{Mg}$ atoms and the slightly corrugated $\mathrm{B} / \mathrm{C} 6^{3}$ nets. The short B-C contacts within the nets, $1.56-1.60$ $\AA$, are certainly within bonding range and can thus be compared to $\mathrm{B}-\mathrm{C}$ distances in other compounds with $\mathrm{B} / \mathrm{C}$ layers, for example, $\mathrm{CaB}_{2} \mathrm{C}_{2}{ }^{7}$ These observed distances are somewhat shorter compared to a value of $1.62 \AA$ expected for a B-C single bond, ${ }^{8}$ suggesting the presence of some $\pi$-bonding character within the hexagonal linkage. The $\mathrm{B}$ and $\mathrm{C}$ distances to the $\mathrm{Mg}$ atoms in this structure vary from 2.25 to $2.57 \AA$ and fall in typically ionic ranges, and so we adopt a Zintl viewpoint, viewing this as $\left(\mathrm{Mg}^{2+}\right)\left(\mathrm{B}_{2} \mathrm{C}_{2}\right)^{2-}$. Here it is assumed that the electropositive magnesium atom is only acting as a two-electron donor with respect to the $\mathrm{B}_{2} \mathrm{C}_{2}$ net. The graphite-like layers of $\mathrm{B}$ and $\mathrm{C}$ in $\mathrm{MgB}_{2} \mathrm{C}_{2}$ are alternating so that $\mathrm{B}$ is nearest neighbor to $\mathrm{C}$ along the $c$ axis as well as within the layer, as shown in Figure 1. Ignoring the $\mathrm{Mg}^{2+}$, what we see is a series of double layers of $\mathrm{B}_{2} \mathrm{C}_{2}{ }^{2-}$, (a)

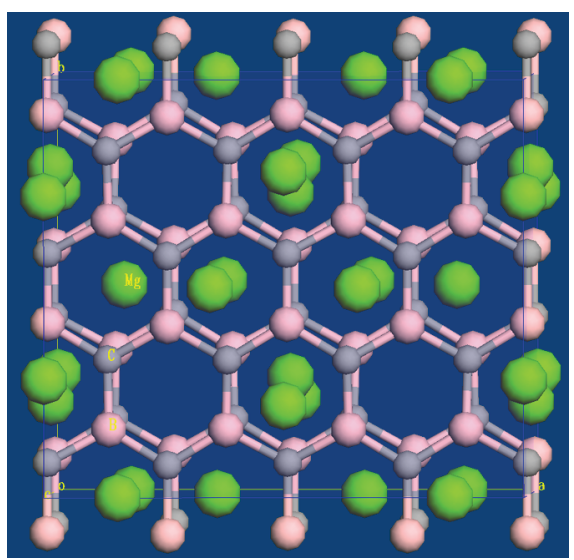

(b)

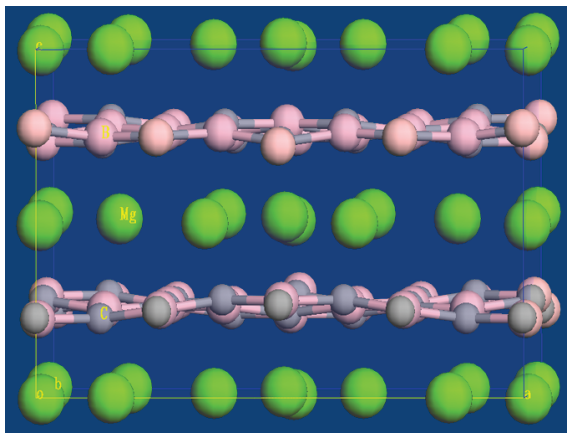

Figure 1. Crystal structure of $\mathrm{MgB}_{2} \mathrm{C}_{2}$ : (a) (001) view of the $a b$ plane, (b) (010) view of the $a c$ plane.

repeating in the $c$ direction. All the layers are parallel, giving the shortest layer-layer separations of $3.74 \AA$.

\section{Results and Discussion}

We begin our discussion with the $2 \mathrm{D} \mathrm{B} / \mathrm{C}$ networks appropriate for $\mathrm{MgB}_{2} \mathrm{C}_{2}$. To a first approximation, we may consider $\mathrm{Mg}$ merely as a two-electron donor and ignore the possible role of magnesium atoms for the time being. Since any two $\mathrm{B}_{2} \mathrm{C}_{2}{ }^{2-}$ layers are so far apart, we may neglect the layer-layer interactions and simplify our calculations by employing twodimensional single $\mathrm{B}_{2} \mathrm{C}_{2}{ }^{2-}$ layer models. It actually can be checked by calculating electronic structures for both single and double layers. The density of states (DOS) plots calculated for these layers are shown in Figure 2. The most important difference is that the band number in Figure $2 b$ is doubled as compared with that in Figure 2a. The resemblance of the bands between them is remarkable, and it is clearly sufficient to use the single layer models in the calculations.

A series of model calculations was carried out using an ideal graphite network for three coloring configurations. A common bond distance of $1.58 \AA$ and regular hexagonal rings were used. The atomic parameters used are not real ones for boron and carbon atoms (see Appendix). While in coloring I all distances are close to the experimentally observed values, in II and III the B-B and C-C bond lengths are not as appropriate. In order to overcome this problem, we performed the 
(a)

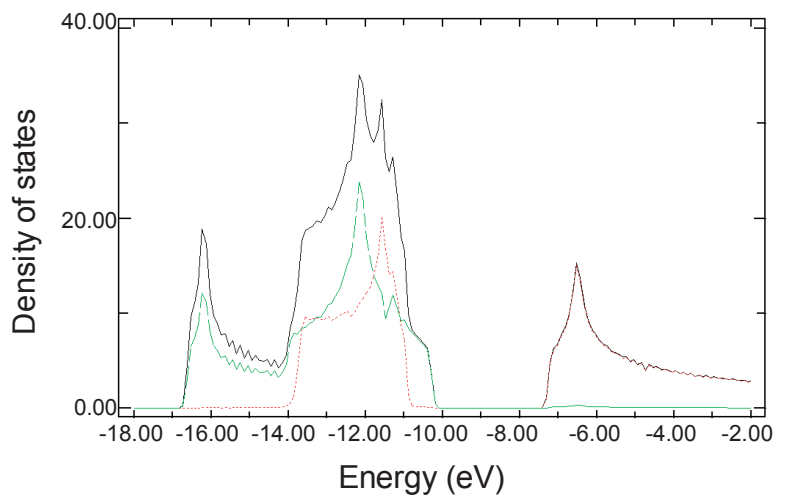

(b)

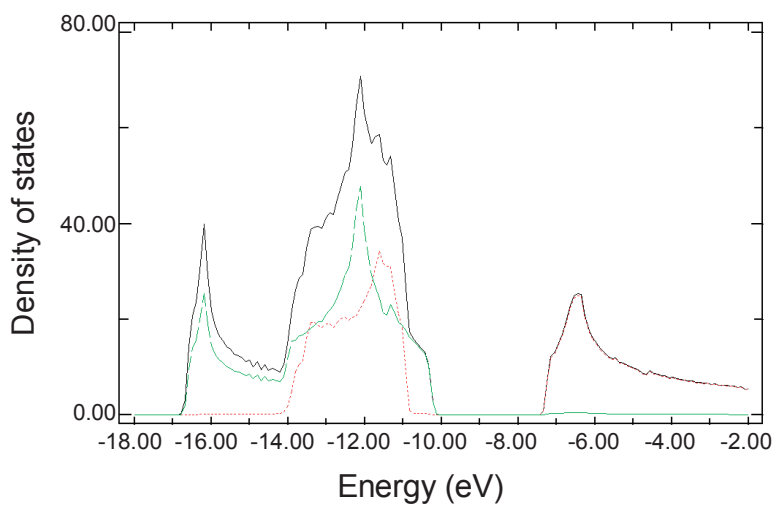

Figure 2. DOS curves calculated for the $\mathrm{B}_{2} \mathrm{C}_{2}$ (a) single and (b) double layers. The $p_{z}$-based $\pi$ contribution to the total DOS (solid line) is indicated as red dotted line and the $p_{x, y}$-based $\sigma$ contribution is indicated as green dashed line.

calculations using the ideal network built from the common distance and the same exponents $\left(\zeta_{2 \mathrm{~s}, 2 \mathrm{p}}=1.30\right)$ for all atoms. The three colorings were simulated with different electronegativities (i.e., different values of $\mathrm{H}_{\mathrm{ii}}$ 's) for the two atoms.

Electronic structure and stability of the $\mathrm{B}_{2} \mathbf{C}_{2}{ }^{2-}$ layer. Let us consider the electronic structure of 2D models made up of a single $\mathrm{B}_{2} \mathrm{C}_{2}{ }^{2-}$ layer. One source of interest in this system is a choice that is made by the $\mathrm{B}$ and $\mathrm{C}$ atoms in occupying hexagonal sublattice sites, a general aspect of isomerism in the solid state. The unit cell of $\mathrm{a}_{2} \mathrm{C}_{2}{ }^{2-}$ layer contains four atoms, two of which are borons, and the other two are carbons. Several ways are possible to accommodate these boron and carbon atoms in the six-membered ring of the $6^{3}$ net. Depicted in 1 are the three models which we denote as coloring I, II, and III. Arrows indicate the unit cell vectors. Coloring I is the experimentally observed structure with alternating $\mathrm{B}$ and $\mathrm{C}$ atoms. It is not obvious why it was chosen as an experimentally observed structure, or what in fact is the order of thermodynamic stability of these isomeric lattices. Question of structural preferences, or "coloring problem" arises frequently in chemistry. In order to approach the coloring problem in this case, we can use the $\mathrm{BC}$ or $\mathrm{B}_{2}$ and $\mathrm{C}_{2}$ units as a starting point and build up the electronic structure of the $\mathrm{B}_{2} \mathrm{C}_{2}{ }^{2-}$ isomers by assembling these in different ways (see 1).

We start our analysis by first looking at the electronic structure of coloring I with a unit cell containing two $\mathrm{BC}^{-}$units. The $\mathrm{B}-\mathrm{C}$ bond lengths are chosen as $1.58 \AA$. This value is the average (a)

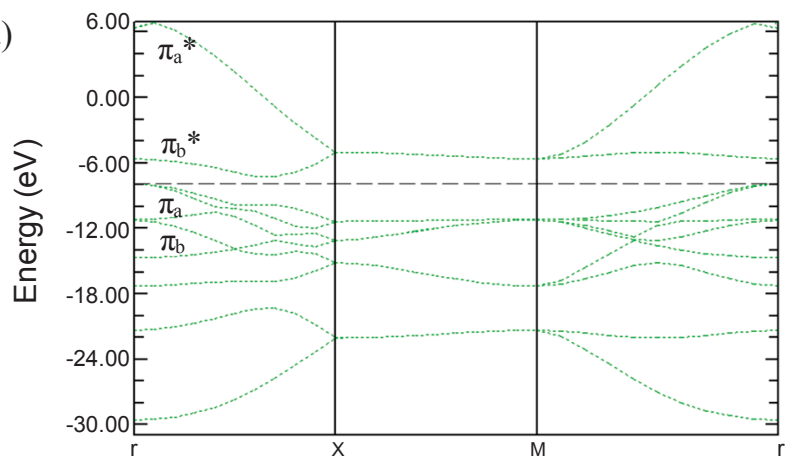

(b)

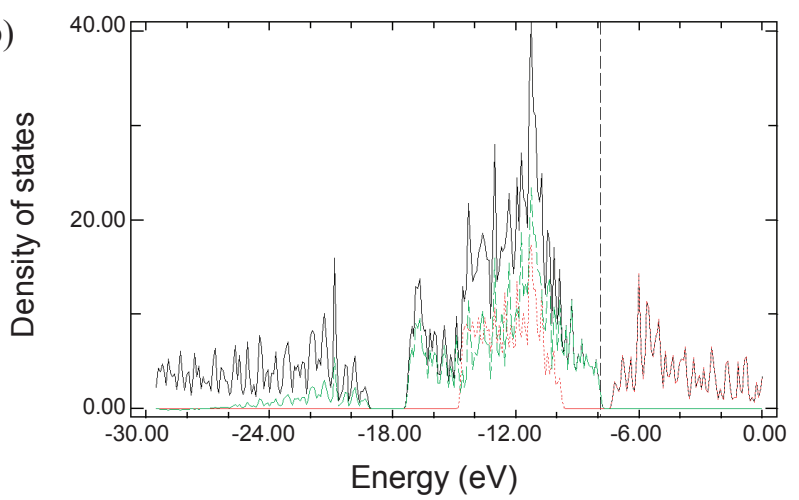

(c)

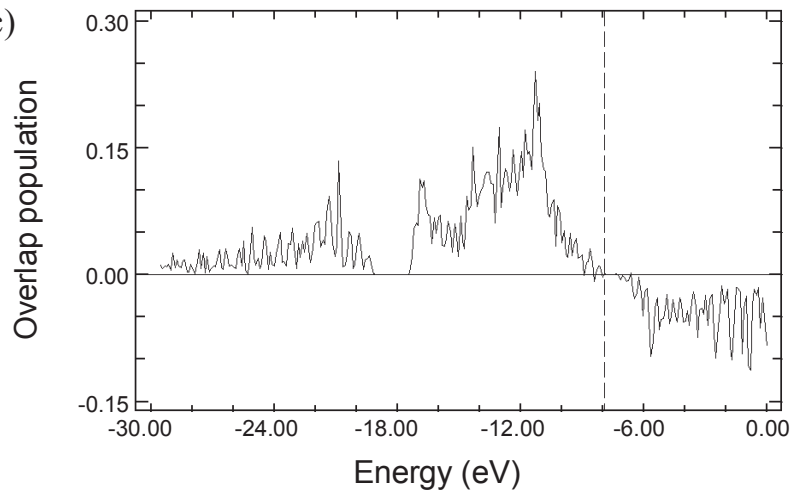

Figure 3. Band structure, DOS, and COOP curves for coloring I of the $\mathrm{B}_{2} \mathrm{C}_{2}{ }^{2-}$ layer: (a) band structure; (b) DOS, the $p_{z}$-based $\pi$ contribution to the total DOS (solid line) is indicated as red dotted line and the $p_{x, y^{-}}$ based $\sigma$ contribution is indicated as green dashed line; (c) COOP curve for the B-C bond. The dashed line indicates the Fermi level.

of the different B-C bond lengths within the nets of the full crystal structure. The calculated band structure and DOS plots for the net associated with the $\mathrm{B}_{2} \mathrm{C}_{2}$ unit cell are shown in Figure 3, a and b, respectively. As can be seen, there is a sizable gap $(0.6 \mathrm{eV})$ for the occupation corresponding to $\mathrm{B}_{2} \mathrm{C}_{2}{ }^{2-}$. The lower and upper two bands nearest to the Fermi level represent $\sigma$ and $\pi^{*}$ bands, respectively, which are based on the in-plane $p_{x, y}$ orbitals and the out-of-plane $p_{z}$ orbitals. These $\sigma$ and $\pi^{*}$ orbitals are the major contributors to the DOS in the region near the Fermi level. The second and third groups of $\pi$ bands, $\pi$ and $\pi^{*}$, repel along $\Gamma \rightarrow X$. However, their wave functions do effectively cross, by which the bands change character as if their lines had actually crossed.

The $\pi$-type orbitals are indicated at the $\Gamma$ point. The two low- 
est occupied $\pi$ bands can be unambiguously associated with the B-C $\pi$ bonding states, whereas the two upper empty $\pi$ bands correspond to the B-C $\pi$ antibonding states. The bonding $\pi_{\mathrm{b}}$ and $\pi_{\mathrm{a}}$ bands are preferentially localized on the more electronegative carbon atoms, while the antibonding $\pi_{\mathrm{b}}{ }^{*}$ and $\pi_{\mathrm{a}}{ }^{*}$ bands just above the gap are mainly localized on the less electronegative boron atoms, because the $2 p$ orbital energies are lower for $\mathrm{C}$ than for $\mathrm{B}$. The subscripts $\mathrm{b}$ and a stand for bonding and antibonding between the two $\mathrm{BC}^{-}$units, respectively. With the electron counting for $\mathrm{B}_{2} \mathrm{C}_{2}{ }^{2-}$ only two of the four $\pi$ bands should be fully occupied. Notice the sizable pseudo band gap $(2.5 \mathrm{eV})$ which separates the $\pi\left(6^{\text {th }}\right)$ band at $1.9 \mathrm{eV}$ below the Fermi level and the $\pi^{*}\left(9^{\text {th }}\right)$ conduction band in Figure $3 \mathrm{a}$. The overlap between these bands for colorings II and III in Figure 4, a and $\mathrm{b}$, results in the disappearance of the gap. In fact, the crossing of the second and third $\pi$ bands in colorings II and III lies at the heart of the instability of their structures. As a result, our calculations indicate that the most stable structure (i.e., coloring I) should be an electrical semiconductor, in good agreement with the observation. The as yet unsynthesized isomers might be metallic.

It is interesting to note that it is only for coloring I that a gap develops at the Fermi level, and this fact is clearly related to the stability of this system, i.e., it is the observed one. In molecules the presence of a good HOMO-LUMO gap is often used as an indicator of their electronic stability. Similarly, in an extended system the highest occupied band will be important in setting the Fermi energy and geometry preferences. An earlier theoretical study of the $2 \mathrm{D}\left(\mathrm{B}_{2} \mathrm{C}_{2}\right)^{2-}$ network by Burdett et al. ${ }^{5}$ found that the structure for which there is no gap at the Fermi level is unstable with respect to the structure for which a significant band gap is opened at the Fermi level. Interestingly, the preferred arrangement is that in which two boron atoms are further apart rather than adjacent. The same conclusion was observed for $\mathrm{MB}_{2} \mathrm{C}_{2}$ and $\mathrm{MB}_{2} \mathrm{C}_{4}$ compounds. ${ }^{9,10}$

In coloring I both $\pi$ bands are filled but the two $\pi^{*}$ bands are empty, and so the fact that there is a large splitting among them is likely to affect the total energy. The isomer stability order should follow the stabilization of $\pi_{\mathrm{a}}$ band. The valence band that is entirely or mostly filled has a Fermi energy of -7.90 , -7.51 , and $-7.58 \mathrm{eV}$ for coloring I, II, and III, respectively. Colorings II and III have higher Fermi energies and would be unstable relative to coloring I. The calculated total energy per unit cell (Table 2) confirms the expectations that coloring I should be more stable than III, which in turn should be more stable than II. The extra stability of coloring I over III can be understood also from another point of view. The band corresponding to the bonding combinations of the $\mathrm{BC}^{-} \pi^{*}$ orbitals, $\pi_{\mathrm{b}}{ }^{*}$, is filled substantially in colorings II and III but is empty in I. The filling of an antibonding orbital in a molecule, or of an antibonding band in a solid, should result in an increase in energy, and consequently a destabilization of the structure.

Another way to probe the bonding in extended structures is to construct crystal orbital overlap population (COOP) curves. These bonding indicators are really overlap population weighted density of states. Figure 3 shows the band structure, the $\pi$-orbitals contribution to the total DOS, and the B-C COOP for coloring I. Note the $\mathrm{B}-\mathrm{C}$ bonding in the lower occupied bands and the (a)

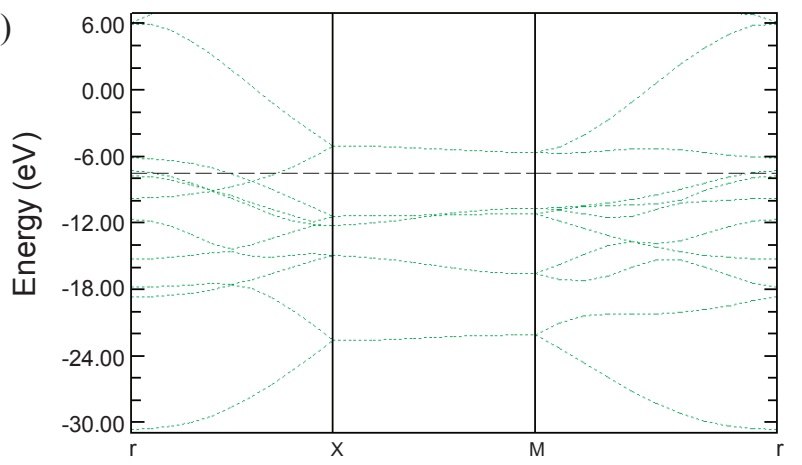

(b)

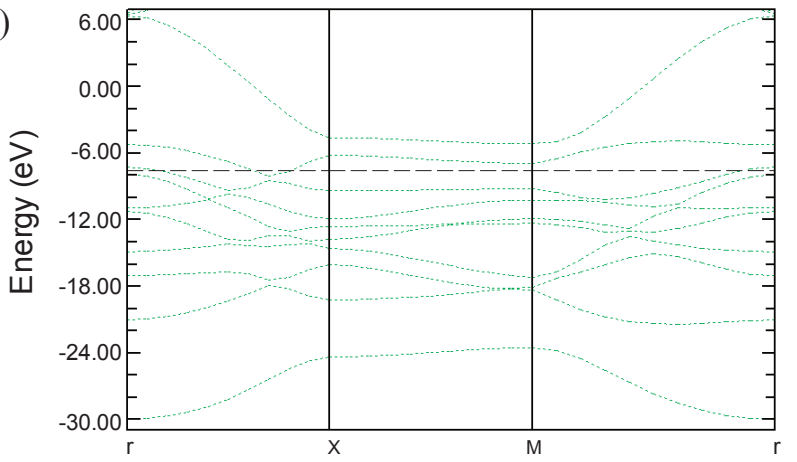

Figure 4. Band structure for (a) coloring II and (b) coloring III of the $\mathrm{B}_{2} \mathrm{C}_{2}{ }^{2-}$ layer. The black dashed line indicates the Fermi level.

Table 2. Relative total energy (eV) per unit cell for all three coloring configurations

\begin{tabular}{cccc}
\hline \multirow{2}{*}{ structure } & \multicolumn{3}{c}{ configuration } \\
\cline { 2 - 4 } & $\mathrm{I}$ & $\mathrm{II}$ & $\mathrm{III}$ \\
\hline $\mathrm{B}_{2} \mathrm{C}_{2}{ }^{2-}$ & 0 & 3.44 & 1.77 \\
\hline
\end{tabular}

B-C antibonding in the upper empty bands. Clearly, we see that the heteronuclear interactions are optimized because the Fermi level occurs at the energy where the overlap population for these bonds crosses from bonding to antibonding. This is typical of a covalent bond and is expected from our previous discussion.

Electronic structure of the full $\mathrm{MgB}_{2} \mathrm{C}_{2}$ structure. We need to look at the effect of the metallic environment on the boroncarbon layers with the analysis of the electronic structure of an $\mathrm{MgB}_{2} \mathrm{C}_{2}$ slab. The total DOS and the metal and nonmetal contributions to it are illustrated in Figure 5a. The role of $\mathrm{Mg}$ in $\mathrm{MgB}_{2} \mathrm{C}_{2}$ can be elucidated by completely removing the $\mathrm{Mg}$ atoms from the lattice and repeating the calculations for a hypothetical structure with only B and C atoms. The calculated electronic structure for the $\mathrm{B} / \mathrm{C}$ network alone is given in Figure $5 \mathrm{~b}$ along with that of $\mathrm{MgB}_{2} \mathrm{C}_{2}$. Interestingly, the bands are not deformed appreciably when we remove $\mathrm{Mg}$ from $\mathrm{MgB}_{2} \mathrm{C}_{2}$, indicating that the electrons from $\mathrm{Mg}$ atoms mainly give a shift in $\mathrm{E}_{\mathrm{f}}$ of the boron-carbon sublattice. This viewpoint is confirmed from the DOS (Figure 5), which shows that the topology of the DOS profiles with and without $\mathrm{Mg}$ atoms in $\mathrm{MgB}_{2} \mathrm{C}_{2}$ is almost the same. A very weak interaction between the metal 
(a)

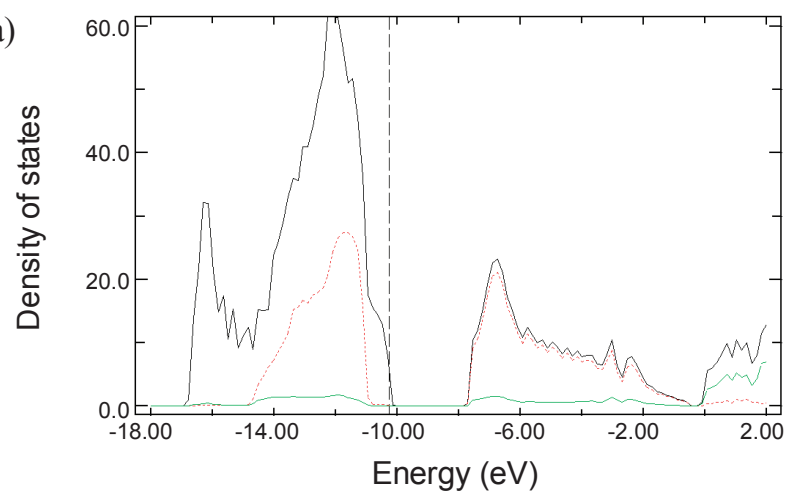

(b)

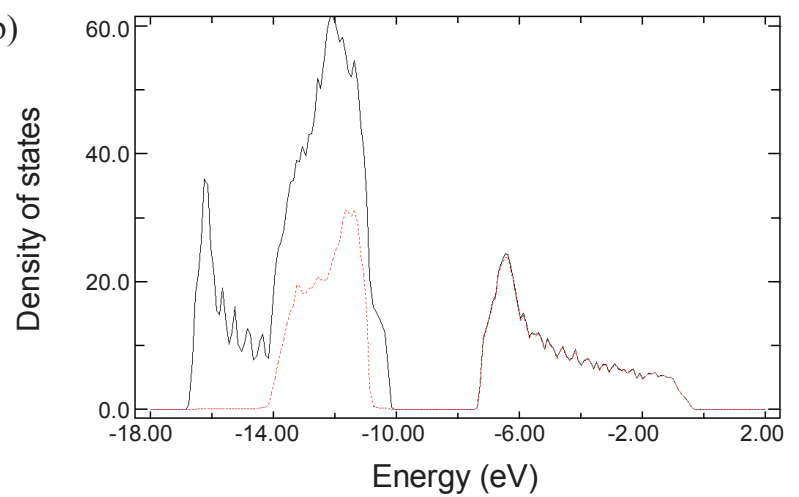

Figure 5. (a) Total DOS (solid line), B-C $\pi$ contribution (red, dotted line), and metallic contribution (green, dashed line) for the $3 \mathrm{D} \mathrm{MgB}_{2} \mathrm{C}_{2}$. The dashed line at $-10.26 \mathrm{eV}$ indicates the Fermi level. (b) Total DOS (solid line) and $\mathrm{B}-\mathrm{C} \pi$ contribution (red, dotted line) for the boroncarbon sublattice before metal-nonmetal interaction in $\mathrm{MgB}_{2} \mathrm{C}_{2}$.

and nonmetal networks leads to the slight downward shift of the BC $\pi$ and $\pi^{*}$ bands compared to $\sigma$ bands, which is due to stronger interaction of nonmetal $2 p_{z}$ orbitals with ionized magnesium sublattice compared to $2 p_{x, y}$ orbitals. A small metallic contribution is present in the region derived mainly from the $\pi$ and $\pi^{*}$ (see Figure 5a). This mixing reflects some covalent character in the bonding between the two sublattices. The topmost valence band is separated from the bottom-most conduction band by an indirect band gap of $2.5 \mathrm{eV}$. It is apparent from the occurrence of the gap that the material is an insulator which is in agreement with earlier studies of Ravindran et al. ${ }^{11}$ and Harima. ${ }^{12}$ The $2 p_{x}$ and $2 p_{y}$ orbitals of $\mathrm{B}$ and $\mathrm{C}$ mainly contribute to the density of states at the top of the valence band, whereas $\mathrm{Mg}$ provides very little contribution to the total DOS at this energy. Consequently, $\mathrm{Mg}$ atoms must be of marginal importance on favoring one or the other coloring. Essentially, they must act as two-electron donors with respect to the boron-carbon network.

Hole doping of $\mathrm{MgB}_{2} \mathrm{C}_{2}$. Band structure calculations indicate that the opening of a gap at the Fermi level is driven by the observed $\mathrm{B}-\mathrm{C}$ alternation in the planes of $\mathrm{B} / \mathrm{C}$ networks. The gap in $\mathrm{MgB}_{2} \mathrm{C}_{2}$, evident in the DOS plots in Figure 5a, lies between $\sigma$ and $\pi_{\mathrm{b}}{ }^{*}$ bands. For the $\sigma\left(2 p_{x, y}\right)$ bands, the pronounced dispersion of $\mathrm{E}(k)$ is observed along the $k_{x}$ and $k_{y}$ directions ( $\Gamma-\mathrm{X}$ and $\Gamma-Y$ of the Brillouin zone in Figure $6 a)$. These bands have a quasi-two-dimensional (2D) character, since they form a flat zone along $k_{z}(\Gamma-\mathrm{Z})$. In contrast to $\mathrm{MgB}_{2}$, they are fully occupied (a)

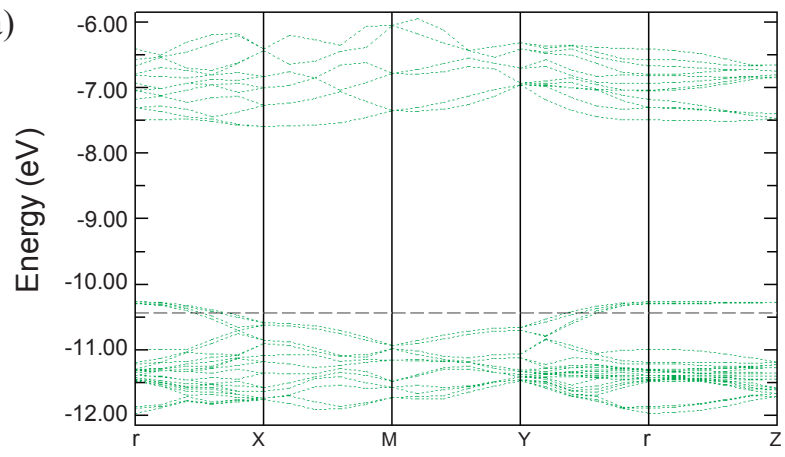

(b)

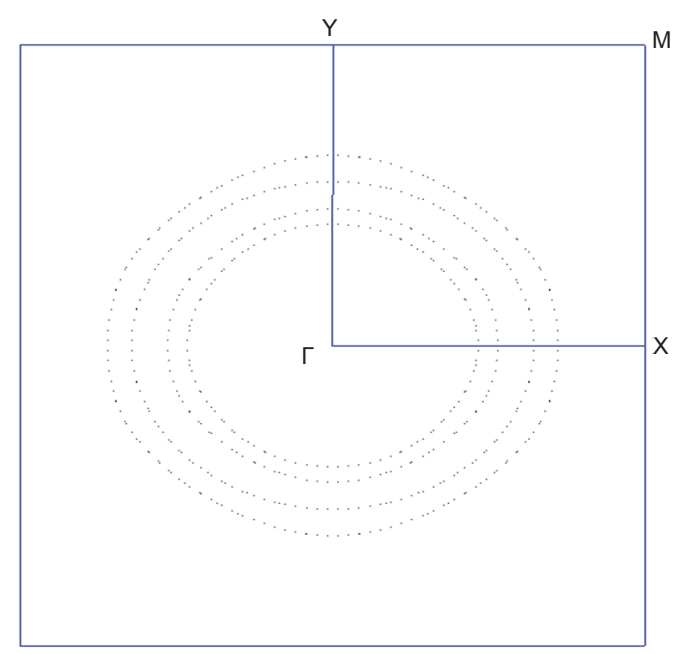

Figure 6. (a) Band structure and (b) Fermi surface for the $3 \mathrm{D} \mathrm{MgB}_{2} \mathrm{C}_{2}$. The Fermi level is indicated for an electron count of $\mathrm{Mg}_{0.875} \mathrm{Li}_{0.125} \mathrm{~B}_{2} \mathrm{C}_{2}$. $\Gamma, \mathrm{X}, \mathrm{M}$, and $\mathrm{Y}$ refer to the wave vectors $(0,0,0),(\mathrm{a} * / 2,0,0),\left(\mathrm{a}^{*} / 2, \mathrm{~b} * /\right.$ $2,0)$, and $\left(0, b^{*} / 2,0\right)$, respectively.

as occurs in LiBC. ${ }^{2}$ By substituting divalent $\mathrm{Mg}$ with a suitably chosen $\mathrm{Li}$ atom, holes are introduced in the $\sigma$ bands. The Lidoped compound $\mathrm{Mg}_{0.875} \mathrm{Li}_{0.125} \mathrm{~B}_{2} \mathrm{C}_{2}$ drives the hole doping of the $\mathrm{B}-\mathrm{C} \sigma$ bonding states in the top of the valence bands. Thus, the 2D Fermi surface of the $\sigma$ bands, which is relevant in the superconductivity in $\mathrm{MgB}_{2}$, was obtained by the hole doping (see Figure 6b). An interesting section of the band structure is the area surrounding $\Gamma$ where an elliptical hole pocket is observed as the highest occupied band rises above the Fermi energy. If the $\sigma$ bands can be populated by a sufficient density of holes, then by analogy with $\mathrm{MgB}_{2}$ the system would be expected to become superconducting. An and Pickett ${ }^{6}$ maintain that the $2 \mathrm{D}$ feature of the $\mathrm{B} \sigma 2 p$ bands is playing an important role in the superconductivity of $\mathrm{MgB}_{2}$. In $\mathrm{MgB}_{2}$, the incompletely filled bonding $\sigma$ bands with predominantly boron $p x$ and $p y$ character give rise to nearly cylindrical, hole-like Fermi surfaces of $2 \mathrm{D}$ character around the $\Gamma$ point, indicating that the transport properties are dominated by the hole carriers in the plane containing B atoms. ${ }^{13}$ As the electronic band structures of both materials with a 2D hole-like Fermi surface are quite similar, the hole-doped $\mathrm{MgB}_{2} \mathrm{C}_{2}$ is believed to be a good candidate for the occurrence of high-temperature superconductivity. 


\section{Concluding Remarks}

In $\mathrm{MgB}_{2} \mathrm{C}_{2}$, three coloring possibilities for the $\mathrm{B}_{2} \mathrm{C}_{2}{ }^{2-}$ net have been theoretically examined. Our electronic band calculations find the alternate $\mathrm{B}$ and $\mathrm{C} 6^{3}$ network to be preferred, crystallographic analysis realizing the borocarbide net. The calculations also reveal that the $\mathrm{B}-\mathrm{C}$ in-plane alternation is responsible for the semiconducting behavior. There are no corresponding gaps in the electronic structure of the $\mathrm{BC}$ layers in which $\mathrm{B}$ and $\mathrm{C}$ do not alternate. In other words, the existence of a band gap explains the higher stability of the coloring I with respect to the other coloring models. We have found that once the parent insulating $\mathrm{MgB}_{2} \mathrm{C}_{2}$ is hole doped, the states at the Fermi level include $\sigma$ bands with elliptical 2D Fermi surfaces of $\mathrm{B}-\mathrm{C} \sigma 2 p$ character. This feature shows that hole-doped $\mathrm{MgB}_{2} \mathrm{C}_{2}$ might be a favorable candidate for superconductivity in $\mathrm{MgB}^{2-}$ like compounds.

Acknowledgments. This work was supported by the Kyungsung University Research Grant in 2010.

Appendix. The results for the ideal nets for I, II, and III reported in Figures 3 and 4 were obtained with use of a common distance of $1.58 \AA$, the same exponents $\zeta_{2 \mathrm{~s}}$ and $\zeta_{2 \mathrm{p}}$ of 1.30 , and the $\mathrm{H}_{\mathrm{ii}}$ values (Table 1) for $\mathrm{B}$ and $\mathrm{C}$.

\section{References}

1. Nagamatsu, J.; Nakagawa, N.; Muronaka, T.; Zenitani, Y.; Akimitsu, J. Nature 2001, 410, 63.

2. Rosner, H.; Kitaigorodsky, A.; Picket, W. E. Phys. Rev. Lett. 2002, $88,127001$.

3. Wörle, M.; Nesper, R. J. Alloys Compd. 1994, 216, 75.

4. (a) Spano, E.; Bernasconi, M.; Kopnin, E. Phys. Rev. B 2005, 72, 014530. (b) Verma, A. K.; Modak, P.; Gaitonde, D. M.; Rao, R. S.; Godwal, B. K.; Gupta, L. C. Europhys. Lett. 2003, 63, 743. (c) Mori, T.; Takayama-Muromachi, E. Curr. Appl. Phys. 2004, 4, 276 .

5. (a) Burdett, J. K.; Lee, S.; McLarnan, T. J. J. Am. Chem. Soc. 1985, 107, 3083. (b) Burdett, J. K.; Canadell, E.; Hughbanks, T. J. Am. Chem. Soc. 1986, 108, 3971.

6. (a) An, J. M.; Pickett, W. E. Phys. Rev. Lett. 2001, 86, 4366. (b) Kortus, J.; Mazin, I. I.; Belashchenko, K. D.; Antropov, V. P.; Boyer, L. L. Phys. Rev. Lett. 2001, 86, 4656. (c) Mazin, I. I.; Antropov, V. P. Physica C 2003, 385, 49.

7. Albert, B.; Schmitt, K. Inorg. Chem. 1999, 38, 6159.

8. Pauling, L. The Nature of the Chemical Bond; Cornell University Press: Ithaca, New York, 1960.

9. Rocquefelte, X.; Boulfelfel, S. E.; Yahia, M. B.; Bauer, J.; Saillard, J.-Y.; Halet, J.-F. Angew. Chem. Int. Ed. 2005, 44, 7542.

10. Fang, C.-M.; Bauer, J.; Saillard, J.-Y.; Halet, J.-F. J. Solid State Chem. 2007, 180, 2465.

11. Ravindran, P.; Vajeeston, P.; Vidya, R.; Kjekshus, A.; Fjellvag, H. Phys. Rev. B 2001, 64, 224509.

12. Harima, H. Physica C 2002, 378-381, 18.

13. Hirsch, J. E. Phys. Lett. A 2001, 282, 392. 